\title{
Is all processed food harmful?
}

\section{What are processed foods?}

Processed food has a very bad reputation as it is blamed to play a vital role in our nation's increasing rates of obesity, hypertension and Type II diabetes. But sometimes, processed foods are advantageous and not all of them are bad. However, industrial processing can mean large amount of salt, sugar and fat which is really bad for our health. Processed foods are not just microwave meals, but the term applies to any kind of food that has been altered from its natural state either for convenience or for increasing its shelf life or to reduce post-harvest losses.

Processed food is basically cooked, heated, baked, salted, canned, frozen or packaged to change its nutritional composition. In simple words, any time we are cooking, baking or preparing food, we are actually processing it. Obviously, most foods we eat are processed in some way or other. Ingredients such as salt, sugar and fat sometimes are added to make the flavor more appealing or to help give it longevity that can lead to weight gain, diabetes and high blood pressure.

\section{Homemade, junk and healthy processed food}

The advantage of cooking food from scratch at home is that you know what is exactly going into it and you can also control the amount of salt, sugar or fat. Moreover, many processed foods contain ingredients that are not actually food, but are just chemicals added for different purposes and we cannot find the clue of many ingredients like emulsifiers, stabilizers \& E coded chemicals that are usually colors or preservatives. Even home-made food sometimes contains processed ingredients. But, processed food is more than margarines, ketchup, crisps or sausages that are not pure but amalgamations of various products. These are just junk processed foods. The whole wheat bread, cheese, canned beans or home-made soups are also processed as they contain processed ingredients but these fall in the category of healthy processed foods. At the same time, they are very affordable and quick to prepare making them highly practical in today's lifestyle.

\section{Categorization of food processing}

Also, there is a difference between mechanical processing and chemical processing. Mechanical processing is no harm to us. For example, ground beef has been ground in a machine and butter is cream that has been separated from the milk and churned to get the final product. If only mechanical processing is done to a food with no added chemical, then it is still the real food and can be beneficial for us and can play an important role in meeting the demands of a balanced diet. On the other hand, when any chemical, whether it be sugar, salt or any preservative is added to the food to improve its taste, color, texture or shelf life, it can be unhealthy for us if consumed on regular basis. For example, BHA (butylated hydroxyanisole) used to preserve potato chips, instant teas, cake mixes etc is expected to cause the liver diseases and cancer. Also, caramel is a coloring agent that causes the vitamin B6 deficiencies, genetic effects and cancer and is mostly found in candies, brown colored food and frozen pizza.
Volume 6 Issue 6 - 2017

\author{
Mateen Anwar,' Zainab Hussain ${ }^{2}$ \\ 'Food Technologist, Quality \& Food Safety Auditor, Pakistan \\ ${ }^{2}$ Tabba Heart Institute (THI), Pakistan
}

Correspondence: Mateen Anwar, Food Technologist, Quality \& Food Safety Auditor, Baskin Robbins, Riyadh, KSA, Pakistan, Email mateenanwer@yahoo.com

Received: June 21, 2017 | Published: July 10, 2017

\section{Processing V/S Fortification}

Not all processed foods are bad. While you should be weary of the term fortified, some processing of food is necessary to prevent illness. However, technically the term can also be used synonymously with fortified foods that have additional nutrients added to make them more healthy and safer such as milk, which needs to be pasteurized and sometimes skimmed to remove harmful bacteria while raw milk can cause food borne illness due to presence of Escherichia Coli, Salmonella and Listeria. Milk and juices are also processed (fortified) with calcium and Vitamins while breakfast cereals may have added fiber that helps in providing bulk to the stool. High fiber foods may have other heart-health benefits, such as reducing blood pressure and inflammation, reducing the risk of developing type II diabetes and aiding in achieving healthy weight. Moreover, processing food is the only way that vegetarians can get Vitamin B12 in their diet. Example of processed foods with added Vitamin B12 includes rice and soy beverages, ready-to-eat breakfast cereals etc.

\section{Processing relevant to fruits $\&$ vegetables}

Frozen fruits and vegetables are promptly blanched, boiled, or steamed, and then frozen within hours of being picked, a process that helps lock in both fresh taste and nutritional value. Frozen produce is also available year-round, and in most cases is cheaper than fresh. But canned fruits and vegetables contain different preservatives and can be unhealthy for us.

\section{Conclusion}

Processed foods are very important as a backup especially in environmental disasters. But excess consumption of processed food has increased because of fast changing life styles, influence of media, increasing urbanization and changing preferences of the population. This demand is further augmented by the availability of a wide range 
of processed foods at a growing number of international and locally owned retail supermarkets in urban areas. Some processed foods are usually loaded with such ingredients like sugar that people get addicted to it and just can't stop eating these foods, no matter how hard they try. Real food is the key to good health, while processed food is not. If consumed in limited amounts, processed foods can give us many benefits and help us in leading a healthy life.

\section{Acknowledgements}

First and foremost, I am grateful to Almighty ALLAH for giving me endurance to this manuscript, for helping me in every single step of this and making it possible. I am thankful to my friend, ZAINAB HUSSAIN, for her encouragement and many fruitful discussions on this article and whose expertise, understanding and generous support enabled me to complete my article without any difficulty. It won't be fair if I don't express my thanks to her as took out her precious time to edit this report and bring in more clarity.

\section{Conflict of interest}

Author declares that there is no conflict of interest. 